\title{
External validation of PADUA classification for the renal tumor enucleation technique
}

Vittori G., Minervini A., Salvi M., Tuccio A., Siena G., Mantella A., Sebastianelli A., Saleh O., Serni S., Carini M.

University of Florence, Dept. of Urology, Florence, Italy

INTRODUCTION \& OBJECTIVES: The PADUA classification was introduced in 2009, but it has never been validated for the Tumor Enucleation (TE) technique. Aim of study was to assess the ability of PADUA score to predict the surgical results and complications of TE.

MATERIAL \& METHODS: We prospectively gathered data from 244 consecutive patients treated with open TE with warm ischemia. All clinical and surgical data, including PADUA score, were registered. The relationship between preoperative variables and surgical results/perioperative complications was assessed with univariate analysis and multivariate logistic regression model.

RESULTS: Median PADUA score of renal tumors resulted 8 (IQR:7-9), and it was $\geqslant 10$ in 52 (21.3\%) patients. Mean warm ischemia time (WIT) was $16.8 \mathrm{~min}$. Overall, 47 perioperative complications occurred in 45 patients (45/244 = 18.4\%); of these, 39 (16\%) were surgical and $8(3.3 \%)$ medical. Surgical complications included blood loss treated with bedrest in 2 patients (0.8\%), with transfusions in $25(10.2 \%)$ and with a second invasive procedure in $5(2.1 \%)$ patients. Urinary fistula occurred in 7 patients $(2.9 \%)$; it was treated with bedrest and antibiotics in $4(1.7 \%)$, while it needed ureteral stenting in the other 3 cases (1.2\%). According to Clavien system, 8 surgical complications were grade 3 ( 3.3\%), and no grade 4 and 5 surgical complications occurred in this series.

At univariate analysis the PADUA score resulted significantly correlated with WIT $(p<0.0001)$, estimated blood loss $(p=0.003)$ and operative time $(p=0.027)$. Significant predictors of overall surgical complications resulted the Charlson index $(p<0.0001)$, the surgical indication $(p=0.045)$, the tumor diameter $(p=0.042)$ and the PADUA score $(p=0.009)$. At the multivariate analysis, independent predictors of overall perioperative complications resulted PADUA score $(R R=1.54 ; p=0.017)$ and the surgical indication $(R R=2.59 ; p=0.019)$. Therefore, each point increase in PADUA score was associated with a 1.54-fold increase in the risk of complications after TE. The PADUA score was the only anatomical variable who independently predict the surgical complications $(R R=1.58 ; p=0.016)$, and Clavien grade III surgical complications (RR=2.99; $p=0.008)$.

CONCLUSIONS: The PADUA score was significantly associated with the surgical results of $\mathrm{TE}$, and it resulted an independent predictor of overall complications, surgical complications, and Clavien grade III complications. 